\title{
MECANISMOS INTUITIVO E RACIONAL: VALIDAÇÃO DE ESCALA PARA TOMADA DE DECISÃO
}

\author{
INTUITIVE AND RATIONAL MECHANISMS: \\ VALIDATION OF SCALE FOR DECISION-MAKING
}

Anna Cecília Chaves Gomes

Instituto Federal da Paraíba - Brasil.

anna.gomes@ifpb.edu.br

Anderson Luiz Rezende Mól

Submissão: 13/02/2019. Aceitação: 0|/102019.

Universidade Federal do Rio Grande do Norte - Brasil mol@ufrnet.br

Publicação: 30/12/2019. Sistema de avaliação: Double

blind review. Universidade FUMEC / FACE, Belo Horizon-

te - MG, Brasil. Editores Prof. Dr. Mário Teixeira Reis Neto

Mayara Carla Marques

Universidade Federal do Rio Grande do Norte - Brasil mayaracarlamarques@gmail.com

\section{RESUMO}

Embora exista significativa aceitação da estrutura tripla por estudos da inteligência artificial, a literatura atual converge para um processamento duplo. Dessa forma, necessita-se ainda de discussões acerca dos componentes da mente. Sendo assim, o presente trabalho propõe validar uma escala para mensuração dos Sistemas Cognitivos, aprofundando o entendimento sobre suas divisões. Para isso, baseou-se na Rational Experimental Inventory (REI) para mensurar as diferenças individuais nos estilos de pensar, com um total de 2.843 observações. Como resultados, a escala pôde, então, ser validada com 3 fatores de significativa confiabilidade. Percebeu-se que, embora a intuição tenha se agrupado em um fator único, a racionalidade se dividiu em dois fatores. A amostra parece perceber a racionalidade como procedente de dois diferentes mecanismos: um lógico, e um mais profundo e exigente. A REl foi reconhecida estatisticamente encontrando-se 3 fatores, dois relativos à racionalidade e um à intuição, havendo um relacionamento entre as duas racionalidades.

\section{PALAVRAS-CHAVE}

Validação de escalas. Sistemas cognitivos. Sistema intuitivo. Sistema racional. Análise fatorial. 


\section{ABSTRACT}

Although there is significant acceptance of triple structure by artificial intelligence studies, current literature converges to dual processing. In this way, discussions are still needed about the components of the mind.Therefore, the present work proposes to validate a scale for the measurement of Cognitive Systems, deepening the understanding about its divisions.To do so, it relied on the "Rational Experimental Inventory (REI)" to measure individual differences in thinking styles, with a total of 2,843 observations. As results, the scale could then be validated with 3 factors of significant reliability. It was realized that, although intuition has been grouped into a single factor, rationality has been divided into two factors. The sample seems to perceive rationality as coming from two different mechanisms: a logical, and a deeper and more demanding. The REI was statistically recognized by finding three factors, two related to rationality and one to intuition, with a relationship between the two rationalities.

\section{KEYWORDS}

Scale validation. Cognitive systems. Intuitive system. Rational system. Factor analysis.

\section{INTRODUÇÃO}

A proposição de que os processos cognitivos são oriundos dos Sistemas Intuitivo e Racional encontra referências a partir das teorias do processamento duplo (HAMMOND, 1996; SLOMAN, 1996; KAHNEMAN; FREDERICK, 2002; BASEL; BRUHL, 20I3). Diferentes autores têm trabalhado com existência de dois sistemas, esses abordados sob diferentes terminologias, a exemplo de Evans (1984), Reber (1989), Epstein (1994) Levinson (1995), Sloman (1996), Evans e Over (1996), Hammond (1996) e Stanovich e West (2000).

O Sistema Intuitivo seria automático, de baixa capacidade computacional e relativa inconsciência, tratando de processos mais espontâneos e heurísticos, enquanto o Racional conjuga um processamento controlado abordando aspectos relativos à inteligência analítica (STANOVICH; WEST, 2000; KAH-
NEMAN; FREDERICK, 2002; FRANKISH, 2010). Durante o período de crescimento da popularidade das teorias dos dois sistemas, muitas críticas se tornaram presentes (EVANS; STANOVICH, 20I3).

Estudos como Evans (2008; 20I0) e Stanovich $(2004 ; 201 \mathrm{l})$ trazem reflexões contra a visão do Sistema Intuitivo como único, de forma que propõe que esse deva ser denominado processamento do Tipo I, pois a terminologia permitiria que fosse constituído por múltiplos sistemas neurais e cognitivos. Este sistema seria então composto por um múltiplo de sistemas heterogêneos, tratados como o Conjunto de Sistemas Autônomos (The Autonomous Set of Systems - TASS) (STANOVICH, 2004; CARRUTHERS, 2006; STANOVICH, 2009).

Stanovich (2004) critica a forma como a literatura aborda o Sistema Racional, afirmando que existiria uma acentuada 
distinção. Algumas pessoas apresentariam elevada capacidade cerebral (altos resultados em testes de inteligência) e competência de passar de uma tarefa para outra de forma rápida e eficiente. Contudo, isso não as tornam imunes aos vieses, havendo, para esta teoria, outra capacidade envolvida denominada racionalidade $(\mathrm{KAH}$ NEMAN, 2012). Assim, o Sistema Racional (Tipo 2) apresentaria, em sua composição, uma mentalidade algorítmica e uma reflexiva, abrangendo dois níveis de controle em um mesmo sistema (STANOVICH, 2009; STANOVICH;WEST;TOPLAK 20II; EVANS; STANOVICH, 20 I3;VAUGHAN et al., 20I6; PALKOVICS; TAKAC, 20I6). Kahneman (20I2) afirma que é preciso mais estudos para verificar se a distinção entre inteligência e racionalidade pode conduzir às novas descobertas.

Embora a teoria do processamento triplo ganhe destaque recentemente, estudos da inteligência artificial já tratam dos sistemas cognitivos como uma estrutura tripla (SAMUELS, 2005; STANOVICH, 2009; VAUGHAN, et al., 2016). Apesar da aceitação na referida área, a maioria da literatura atual ainda converge para um processamento duplo, de forma que seguem em andamento as discussões a respeito dos componentes da mente (KEREN; SCHUL, 2009; BASEL; BRUHL, 20 I3).

Dado que tais formas distintas de processamento são distinguíveis por sua velocidade, controlabilidade e conteúdo que operam os trabalhos da área procuram ainda trazer uma representação latente à estrutura da decisão humana (KAHNEMAN; FREDERICK, 2002). Partindo do princípio que o conhecimento dessa estrutura auxilia na tomada de decisão ótima, seriam, assim, necessárias mais pesquisas que se aprofun- dem não apenas em sua composição, mas também na influência dos sistemas cognitivos no processo decisório. Desse modo, o presente trabalho se propôs a validar uma escala para mensuração dos Sistemas Cognitivos, aprofundando assim o entendimento de como estes se apresentam.

\section{REFERENCIAL TEÓRICO}

Os termos Sistema I e Sistema 2 foram cunhados por Stanovich eWest (2000) sendo adotados em diversos estudos em seguida (KAHNEMAN; FREDERICK, 2002). Entretanto, anterior a Stanovich e West (2000) diferentes terminologias eram usadas para tratar as teorias de raciocínio baseadas em dois processos (EVANS, 1984; EPSTEIN, 1994; LEVINSON, 1995; SLOMAN, 1996). O Quadro I traz uma síntese das diferentes representações dos sistemas de lógica baseada em dois processos:

Neste aspecto, o Sistema Intuitivo conjuga propriedades de automaticidade e processamentos heurísticos, podendo ser caracterizado como automático, relativamente inconsciente e pouco exigente da capacidade computacional; enquanto o Sistema Racional conjugaria as várias características típicas de um processamento controlado, englobando, assim, processos de inteligência analítica (STANOVICH; WEST, 2000; KAHNEMAN; FREDERICK, 2002; FRANKISH, 20I0). As principais características comumente associadas a cada um dos dois sistemas estão descritas no Quadro 2 e utilizadas para fim de representação dos sistemas no presente estudo.

Evans e Stanovich (2013) informam que, embora seja conveniente passar a impressão que todas as teorias de duplo processo recorram aos mesmos dois processos, a afirmativa não seria verdadeira. Uma corrente 
QUADRO 1 - Autores que trabalharam com uma lógica de dois processos e suas representações

\begin{tabular}{|c|c|c|}
\hline Autor & Sistema 1 & Sistema 2 \\
\hline Sloman (1996) & Associative system & Rule-based system \\
\hline Evans $(1984 ; 1989)$ & Heuristc processing & Analytic processing \\
\hline Evans e Over (1996) & Tacit thought processes & Explict thought processes \\
\hline Reber (1993) & Implicit cognition & Explicit learning \\
\hline Levinson (1995) & Interactional intelligence & Analytic intelligence \\
\hline Epstein (1994) & Experimential system & Rational system \\
\hline Pollock (1996) & Quick and inflexible modules & Intellection \\
\hline Hammond (1996) & Intuitive cognition & Analytical cogniition \\
\hline Klein (1998) & Recognition-primed decisions & Rational choice strategy \\
\hline Johnson-Laird (1983) & Implicit inferences & Explicit inferences \\
\hline Shiffrin e Schneider (1977) & Automatic processing & Controlled processing \\
\hline Posner e Snyder (1975) & Automatic activation & Conscious processing system \\
\hline
\end{tabular}

Fonte: Adaptado de Stanovich e West (2000).

QUADRO 2 - Características dos sistemas Intuitivo e Racional

\begin{tabular}{|c|c|c|}
\hline & Sistema Intuitivo & Sistema Racional \\
\hline \multirow{6}{*}{ 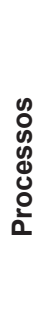 } & Rápido & Lento \\
\hline & Automático & Controlado \\
\hline & Inconsciente ou quase inconsciente & Consciente \\
\hline & Baixo esforço, alta capacidade & Alto esforço, baixa capacidade \\
\hline & Heurístico & Analítico \\
\hline & Associativo & Baseado em regras \\
\hline \multirow{4}{*}{ 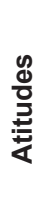 } & Implícito & Explícito \\
\hline & Estereótipos culturais & Crenças pessoais \\
\hline & Aquisição e mudanças lentas & Aquisição e mudanças rápidas \\
\hline & Acesso rápido & Lento acesso \\
\hline \multirow{4}{*}{$\begin{array}{l}\frac{0}{0} \\
\frac{0}{0} \\
\stackrel{0}{0}\end{array}$} & Atual & Hipotético \\
\hline & Concreto & Abstrato \\
\hline & Contextualizado & Descontextualizado \\
\hline & Domínio específico & Domínio geral \\
\hline \multirow{3}{*}{ 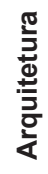 } & Um conjunto de sistemas, modular & Um sistema único \\
\hline & Paralelo & Serial \\
\hline & Não utiliza a memória de trabalho & Usa memória de trabalho \\
\hline \multirow{4}{*}{ 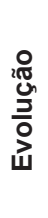 } & Evolutivamente antigo & Evolutivamente recente \\
\hline & Compartilhado com os animais & Único aos humanos \\
\hline & Não verbal & Envolve linguagem \\
\hline & Serve objetivos genéticos & Serve objetivos individuais \\
\hline \multirow{3}{*}{ 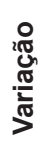 } & Independente da inteligência geral & Relacionado à inteligência geral \\
\hline & Pequenas variações entre culturas e indivíduos & Varia entre culturas e indivíduos \\
\hline & Relativamente não responsável pela introdução verbal & Responsável pela introdução verbal \\
\hline
\end{tabular}

Fonte: Frankish (2010). 
significativa representada pelos psicólogos evolucionistas, por exemplo, acredita que a maioria das respostas do Sistema Intuitivo é adaptável evolutivamente, o que não implica na afirmação (fornecida por muitos pesquisadores de heurísticas e vieses) de que a alternativa é racional ao nível do indivíduo (STANOVICH, 2009). Tal corrente segue em consonância com Stanovich (2009), Stanovich, West e Toplak (20 I I), Evans e Stanovich (20I3), que vieram sugerir uma estrutura tripla. De fato, pesquisadores da inteligência artificial já tratam, de forma robusta, de uma estrutura tripla (SLOMAN; CHRISLEY, 2003; SAMUELS, 2005; STANOVICH, 2009; VAUGHAN, et al., 2016).

Para os pesquisadores da área, o Sistema Intuitivo seria formado por um conjunto de sistemas neurais e cognitivos. Esses seriam heterogêneos e deveriam ser tratados como Conjunto de Sistemas Autônomos (STANOVICH, 2004; CARRUTHERS, 2006 STANOVICH, 2009; EVANS; STANOVICH, 20I3).

Ao se questionar o que faz uma pessoa mais suscetível a vieses que outras, Stanovich (2009) traça ainda uma distinção entre duas partes do Sistema Racional, diferenciando a racionalidade da inteligência. Para o autor, a mente algorítmica seria aquela que trabalha com pensamentos lentos e cálculos exigentes, essa seria ligada à capacidade cerebral e aos testes de inteligência. Já a reflexiva estaria relacionada à racionalidade e abrangeria a tendência com a qual o indivíduo se empenha em parar de pensar; pessoas racionais seriam mais ativas intelectualmente e menos propensas a se satisfazerem com respostas superficialmente atraentes (intuições) (KAHNEMAN, 20I2). A mente algorítmica trata de sustentar representações dissociadas da intuição, enquanto a mente reflexiva opera na detecção da necessidade de encontrar uma alternativa à solução "menos exigente" (STANOVICH,WEST; TOPLAK, 20I I). Para Evans e Stanovich (20I3, p. 230), a mente reflexiva trabalha com:

[...] a tendência para coletar informações antes de fazer-se uma ideia, a tendência para procurar vários pontos de vista antes de chegar a uma conclusão, a disposição para pensar muito sobre o problema antes de responder, a tendência para calibrar o grau de força de sua opinião com o grau de evidência disponível, a tendência para pensar sobre as consequências futuras antes de agir, e a tendência para pesar explicitamente prós e contras de situações antes de tomar uma decisão.

Para Kahneman (2012, p. 65), "só o tempo dirá se a distinção entre inteligência e racionalidade pode conduzir a novas descobertas".

Para fins do presente estudo, adotou-se a divisão dos Sistemas conforme tratado por Stanovich e West (2000) e Kahneman e Frederick (2002) sob as denominações de Sistema Intuitivo (Sistema I) e Sistema Racional (Sistema 2), sem pressupor a existência de nenhuma outra subdivisão.

\section{METODOLOGIA}

Epstein (1994) propõe a Cognitive Experimential SelfTheory (CEST) integrando o cognitivo com a psicodinâmica inconsciente, que é uma teoria acerca do processamento de informações humanas que sugerem a existência de dois sistemas de processamento: o racional e o experimental. Para Sinclair (20I4), as divisões correspondem aos Sistemas Intuitivo e Racional propostos pelos teóricos do processamento duplo.

Tomando como base o CEST, desenvolveu-se o Rational Experimential Inventory (REI) para mensurar as diferenças individuais nos estilos de pensar intuitivo-experi- 
mental e racional-analítico (EPSTEIN et al., 1996). Este é concebido com 31 itens. A escala de pensamento racional foi desenvolvida a partir da escala Need for Cognition (NFC) de Cacioppo e Petty (I982). Já a escala de pensamento intuitivo foi desenvolvida pelos próprios autores e denominada Faith in Intuition (FI), originalmente concebida com 12 questões, visando capturar o quão os indivíduos estão confiantes em utilizar seus sentimentos e impressões imediatas como base na tomada de decisão.

Uma escala de 40 itens da REl foi, subsequentemente, desenvolvida por Pacini e Epstein (1999) subdividindo os sistemas, racional e intuitivo, em subescalas de habilidade e engajamento com uma mistura de itens positivos e negativos.

Hodgkinson et al. (2009) utilizou estudantes australianos e descobriu que a REI seria consistente com a divisão em apenas dois fatores: racional e intuitivos, não conseguindo replicar a estrutura de quatro fatores de Pacini e Epstein (1999). Em outros estudos com estudantes espanhóis e holandês, Witteman et al. (2009) também faIhou em observar os quatro fatores, igualmente observando a existência de apenas dois fatores. Por fim, Akinci e Sadler-Smith (20l3), em um estudo com profissionais, foram identicamente incapazes de replicar a estrutura habilidade/engajamento, observando apenas as duas dimensões básicas com altos níveis de consistência e baixa correlação interescalas. Neste trabalho, a REI foi utilizada em sua versão de 40 itens. As questões foram codificadas e pontuadas de acordo com o Quadro 3.

A escala foi analisada a partir de validação fatorial e o score calculado como a soma dos valores individuais de cada fator oriundo da validação.
Ao todo foram coletadas 2.958 observações, no entanto, após a exclusão de questionários com dados ausentes (os entrevistados não responderam mais de $10 \%$ das perguntas ou que possuíam apenas ensino médio), a amostra reduziu para 2.843 observações.

Para fins da presente pesquisa, utilizouse de uma amostragem não probabilística realizada no segundo semestre de 2015 . Contatou-se todas as universidades federais e estaduais, por e-mail e telefone, através das pró-reitorias de graduação e pós-graduação.

As universidades que se comprometeram a divulgar o questionário foram: Universidade do Estado do Pará (UEPA), Universidade Federal do Sul e Sudeste do Pará (UNIFESSPA), Universidade Federal do Tocantins (UFT), Universidade Federal do Recôncavo da Bahia (UFRB), Universidade Federal da Bahia (UFBA), Universidade Federal do Ceará (UFC), Universidade Federal Rural de Pernambuco (UFRPE), Universidade Federal de Sergipe (UFS), Universidade Federal do Rio Grande do Norte (UFRN), Universidade Estadual do Rio Grande do Norte (UERN), Universidade Federal de Uberlândia (UFU), Universidade Federal do Triângulo Mineiro (UFTM), Universidade Estadual de Campinas (UNICAMP), Universidade Federal Fluminense (UFF), Universidade Federal do Estado do Rio de Janeiro (UNIRIO), Universidade Federal de Goiás (UFG), Universidade do Estado de Mato Grosso (UNEMAT), Universidade Federal de Mato Grosso do Sul (UFMS), Universidade Federal da Grande Dourados (UFGD), Universidade Federal do Rio Grande do Sul (UFRGS) e Universidade Federal de Santa Maria (UFSM).

A amostra é composta, assim, predomi- 
QUADRO 3 - Construção da score do Sistema 1 e Sistema 2

\begin{tabular}{|c|c|c|}
\hline CÓDIGO & DESCRIÇÃO & VALORES \\
\hline REI_R1 & Eu tento evitar situações que requerem pensamentos muito profundos sobre algo & Valores entre 1 e 9 \\
\hline REI_R2 & Eu não sou tão bom em entender problemas complicados & Valores entre 1 e 9 \\
\hline REI_R3 & Eu gosto de desafiar meu intelecto & Valores entre 1 e 9 \\
\hline REI_R4 & Eu não sou muito bom em resolver problemas que requerem análises lógicas cuidadosas & Valores entre 1 e 9 \\
\hline REI_R5 & Eu não gosto de ter que pensar muito & Valores entre 1 e 9 \\
\hline REI_R6 & Eu gosto de resolver problemas que requerem que eu pense arduamente & Valores entre 1 e 9 \\
\hline REI_R7 & Pensar não é minha ideia de uma atividade agradável & Valores entre 1 e 9 \\
\hline REI_R8 & Eu não sou um pensador muito analítico & Valores entre 1 e 9 \\
\hline REI_R9 & Racionalizar cuidadosamente não é um de meus pontos fortes & Valores entre 1 e 9 \\
\hline REI_R10 & Eu prefiro problemas complexos a problemas simples & Valores entre 1 e 9 \\
\hline REI_R11 & Pensar arduamente e por um longo tempo sobre algo me dá pouca satisfação & Valores entre 1 e 9 \\
\hline REI_R12 & Eu não pondero bem sobre pressão & Valores entre 1 e 9 \\
\hline REI_R13 & Eu sou muito melhor em entender coisas logicamente do que a maioria das pessoas & Valores entre 1 e 9 \\
\hline REI_R14 & Eu tenho uma mentalidade lógica & Valores entre 1 e 9 \\
\hline REI_R15 & Eu gosto de pensar em termos abstratos & Valores entre 1 e 9 \\
\hline REI_R16 & Eu não tenho problemas em analisar as coisas cuidadosamente & Valores entre 1 e 9 \\
\hline REI_R17 & Utilizar a lógica normalmente funciona bem para que eu resolva problemas em minha vida & Valores entre 1 e 9 \\
\hline REI_R18 & Saber a resposta sem ter de entender a razão por traz dela é bom o suficiente para mim & Valores entre 1 e 9 \\
\hline REI_R19 & Eu geralmente tenho razões explicáveis e claras para as minhas decisões & Valores entre 1 e 9 \\
\hline REI_R20 & Aprender novos modos de pensar seria muito atraente para mim & Valores entre 1 e 9 \\
\hline REI_I1 & Eu gosto de confiar em minhas impressões intuitivas & Valores entre 1 e 9 \\
\hline REI_I2 & Eu não tenho um senso muito bom de intuição & Valores entre 1 e 9 \\
\hline REI_I3 & $\begin{array}{l}\text { Utilizar meus sentimentos instintivos normalmente funciona bem para eu resolver problemas } \\
\text { em minha vida }\end{array}$ & Valores entre 1 e 9 \\
\hline REI_I4 & Eu confio em minhas impressões & Valores entre 1 e 9 \\
\hline REI_I5 & A intuição pode ser um modo muito útil de resolver problemas & Valores entre 1 e 9 \\
\hline REI_I6 & Eu geralmente sigo meus instintos quando decido um curso de ação & Valores entre 1 e 9 \\
\hline REI_I7 & Eu confio em minhas primeiras impressões sobre as pessoas & Valores entre 1 e 9 \\
\hline REI_I8 & Quando se trata de confiar em pessoas, eu geralmente posso confiar em minhas intuições & Valores entre 1 e 9 \\
\hline REI_19 & Se eu fosse confiar em minhas intuições, eu frequentemente cometeria erros & Valores entre 1 e 9 \\
\hline REI_I10 & Eu não gosto de situações nas quais eu tenha que confiar em minha intuição & Valores entre 1 e 9 \\
\hline REI_I11 & Eu acho que existem momentos nos quais se deve confiar em sua intuição & Valores entre 1 e 9 \\
\hline REI_I12 & Eu penso que é bobagem tomar decisões importantes baseadas em impressões & Valores entre 1 e 9 \\
\hline REI_I13 & Eu não acho que seja uma boa ideia confiar em sua intuição para importantes decisões & Valores entre 1 e 9 \\
\hline REI_I14 & Eu geralmente não dependo de meus sentimentos para me auxiliar a tomar decisões & Valores entre 1 e 9 \\
\hline REI_I15 & $\begin{array}{l}\text { Eu raramente erro quando eu escuto minhas intuições mais profundas para encontrar uma } \\
\text { resposta }\end{array}$ & Valores entre 1 e 9 \\
\hline REI_I16 & Eu não quereria depender de alguém que se descreve como intuitivo & Valores entre 1 e 9 \\
\hline REI_I17 & Meus julgamentos instintivos provavelmente não são tão bons como o da maioria das pessoas & Valores entre 1 e 9 \\
\hline REI_I18 & Eu tendo a utilizar meu coração como guia para minhas ações & Valores entre 1 e 9 \\
\hline REI_I19 & $\begin{array}{l}\text { Eu normalmente sinto quando uma pessoa está certa ou errada mesmo não podendo expli- } \\
\text { car como eu sei }\end{array}$ & Valores entre 1 e 9 \\
\hline REI_I20 & Eu suspeito que meus palpites estão imprecisos na mesma medida em que eles são precisos & Valores entre 1 e 9 \\
\hline
\end{tabular}

Fonte: Adaptado de Pacini e Epstein (1999) 
nantemente, por respondentes do sexo feminino $(57,1 \%)$. No que concerne às áreas de atuação, a maioria $(41,6 \%)$ dos respondentes são de artes, humanas e sociais, e em segundo lugar está Biociências (32,9\%). As respostas foram concentradas em três principais estados: Rio Grande do Norte (30,3\%), Espírito Santo (25,9\%) e Minas Gerais (I0,7\%). A Tabela I demonstra a concentração da amostra por região.

Os dados foram tratados através de Estatísticas Descritivas,Análises Fatoriais Exploratórias (AFE), Análises Fatoriais Confirmatórias (AFC).As Análises Fatoriais Exploratórias foram utilizadas para fins de validação dos instrumentos adotados (REl; TOV-R). Essas foram tratadas, posteriormente, a partir da Análise Fatorial Confirmatória. Todos os pro- cedimentos foram realizados no Statistical Package for the Social Sciences (SPSS) versão 22.

Para a implementação da AFE, considerouse os testes/medidas de ajustamentos descritos no Quadro 4.

O método de extração utilizado foi a Análise de Componentes Principais, já que se objetivou resumir o máximo possível da informação original em um número mínimo de fatores. A rotação foi analisada caso a caso. A Análise Fatorial Confirmatória foi implementada, quanto ao seu ajuste, seguindo os critérios estabelecidos por Marôco (20I4), esses representados no Quadro 5.

Vale salientar que problemas quanto aos testes de $\chi 2$ já são esperados, pois essas medidas devem ser relevadas devido às suas sensibilidades ao tamanho da amos-

TABELA 1 - Amostra obtida por região

\begin{tabular}{l|c}
\hline REGIÃO & PERCENTUAL \\
\hline Norte & $1,3 \%$ \\
\hline Nordeste & $48,9 \%$ \\
\hline Centro-oeste & $1,1 \%$ \\
\hline Sudeste & $37,7 \%$ \\
\hline Sul & $11,1 \%$ \\
\hline
\end{tabular}

Fonte: Elaboração própria.

QUADRO 4 - Critérios e coeficientes exigidos para fim da AFE

\begin{tabular}{|c|c|c|}
\hline Índice & Definição & Solicitado \\
\hline $\begin{array}{l}\text { Kayser-Meyer-Olkin } \\
\text { (KMO) }\end{array}$ & $\begin{array}{l}\text { Indica quão adequada é a aplicação da AFE para o conjunto de dados, } \\
\text { sugerindo a proporção de variância dos itens que pode ser explicada por } \\
\text { uma variável latente. }\end{array}$ & Acima de 0,7 \\
\hline Comunalidade & $\begin{array}{l}\text { Quantia total de variância que uma variável original compartilha com todas } \\
\qquad \text { as outras variáveis incluídas na análise. }\end{array}$ & $\begin{array}{l}\text { Acima de } 0,5 \text {, prefe- } \\
\text { rencialmente acima } \\
\text { de } 0,6\end{array}$ \\
\hline $\begin{array}{l}\text { Teste de Esferacidade } \\
\text { de Bartlett }\end{array}$ & $\begin{array}{l}\text { Teste estatístico da significância geral de todas as correlações em uma } \\
\text { matriz de correlação }\end{array}$ & $p$-valor $<0,05$ \\
\hline $\begin{array}{l}\text { Medida de Adequação } \\
\text { da Amostra (MSA) }\end{array}$ & $\begin{array}{l}\text { Medida calculada para avaliar quão adequada é a aplicação de uma análi- } \\
\text { se fatorial. }\end{array}$ & $M S A \leq 0,50$ \\
\hline Cargas Fatoriais & $\begin{array}{l}\text { Correlação entre as variáveis originais e os fatores, bem como a chave } \\
\text { para o entendimento da natureza de um fator particular. Seu valor ao qua- } \\
\text { drado indicam o percentual da variância em uma variável que é explicado } \\
\text { por um fator. }\end{array}$ & Acima de $\pm 0,50$ \\
\hline Alpha de Cronbach & Medida de confiabilidade que varia de 0 a 1. & Acima de 0,60 \\
\hline
\end{tabular}

Fonte: Adaptado de Hair (2005). 
QUADRO 5 - Critérios para ajuste da AFE

\begin{tabular}{|c|c|c|}
\hline Índice & Definição & Solicitado \\
\hline $\begin{array}{c}X^{Y} ; \\
\text { p-value }\end{array}$ & $\begin{array}{l}\text { Visa medir quão discrepante é a amostra do modelo } \\
\text { teórico }\end{array}$ & Quanto menor melhor; $\mathrm{P}>0,05$ \\
\hline \multirow{4}{*}{$X^{r / g l}$} & \multirow{4}{*}{$\begin{array}{l}\text { Padronização do teste qui-quadrado (sensível ao tamanho } \\
\text { da amostra) a partir da divisão pelos graus de liberdade }\end{array}$} & $>5$ ajustamento mau \\
\hline & & ]2;5] ajustamento sofrível \\
\hline & & ]1;2] ajustamento bom \\
\hline & & $\sim 1$ ajustamento muito bom \\
\hline \multirow{3}{*}{$\begin{array}{l}\text { Comparative Fit } \\
\text { Index (CFI) }\end{array}$} & \multirow{3}{*}{$\begin{array}{l}\text { Corrige a subestimação ao se utilizar o NFI para amostras } \\
\text { pequenas. Compara também o ajustamento do modelo em } \\
\text { estudo }\left(\mathrm{X}^{2}\right) \text { com os graus de liberdade }(\mathrm{gl}) \text { com o ajusta- } \\
\text { mento do modelo basal com os graus de liberdade. }\end{array}$} & [0,8;0,9[ ajustamento sofrível \\
\hline & & {$[0,9 ; 0,95[$ ajustamento bom } \\
\hline & & $\geq 0,95$ ajustamento muito bom \\
\hline \multirow{3}{*}{$\begin{array}{l}\text { Goodness of Fit } \\
\text { Index (GFI) }\end{array}$} & \multirow{3}{*}{$\begin{array}{l}\text { Proporção das covariâncias observadas explicadas pelo } \\
\text { modelo ajustado. }\end{array}$} & {$[0,8 ; 0,9[$ ajustamento sofrível } \\
\hline & & {$[0,9 ; 0,95[$ ajustamento bom } \\
\hline & & $\geq 0,95$ ajustamento muito bom \\
\hline \multirow{2}{*}{$\begin{array}{l}\text { Root Mean Square } \\
\text { Error of Aproxi- } \\
\text { mation (RMSEA); } \\
\quad \text { p-valor }\end{array}$} & \multirow[b]{2}{*}{$\begin{array}{l}\text { Mede a qualidade do ajuste do modelo à matriz de cova- } \\
\text { riâncias da amostra considerando os graus de liberdade }\end{array}$} & ]0,05;0,10] Ajustamento aceitável \\
\hline & & $\begin{array}{l}\leq 0,05 \text { ajustamento muito bom; } \\
\qquad P \text {-valor } \geq \alpha\end{array}$ \\
\hline
\end{tabular}

Fonte: Adaptado de Marôco (2014).

tra (BYRNE, 20I0). Contudo, optou-se por mostrar este resultado dado que os demais o tomariam como base.

Uma vez em que os dados apresentaram falhas de normalidade multivariada, comuns em aplicações nas Ciências Humanas e Sociais, utilizou-se da Distribuição Assimptótica Livre (ADF), que relaxa as pressuposições para normalidade multivariada (MARÔCO, 20l4). Analisou-se, ainda, na AFC a Validade Fatorial, Convergente e Discriminante como propõe Marôco (20|4).A Validade Fatorial ocorre quando as especificações dos itens de um dado constructo estão corretas. Já a Validade Convergente demonstra se os itens constituintes de um constructo apresentam correlações positivas e elevadas entre si. Por fim, a Validade Discriminante ocorre quando o constructo não se encontra, consideravelmente, correlacionado com constructos que operacionalizam variáveis latentes que diferem da operacionalizada pelo constructo. Os três componentes em conjunto demonstram a
Validade de Constructo. Cada qual foi analisado sendo os coeficientes mínimos sugeridos pelo autor para cada tipo de validade destacados no Quadro 6.

\section{RESULTADOS}

De acordo com a Tabela 2, é possível verificar a composição das questões, no que tange a média e desvio padrão, com base nos valores entre I e 9.

Referente ao Sistema 2, a questão que apresentou os valores mais fortes em relação à média diz respeito ao fato de que aprender novos modos seria interessante para as pessoas, sendo a mais próxima do valor máximo, seguida da questão que afirma que utilizar a lógica normalmente funciona bem para que as pessoas resolvam os problemas na vida delas, e ainda a que afirma ter, geralmente, razões explicáveis e claras para as tomadas de decisões. Bem como, o questionamento sobre aprender novos modos de pensar seria muito atraente, também apresentou o menor desvio 
QUADRO 6 - Testes de validade realizados

\begin{tabular}{|c|c|c|}
\hline Tipo de Validade & Índices Utilizados & Valor solicitado \\
\hline Validade Fatorial & Confiabilidade individual () & $>0,25$ \\
\hline \multirow{2}{*}{ Validade Convergente } & Variância Média Explicada (VME) & VME $\geq 0,5$ \\
\cline { 2 - 3 } & Confiabilidade Composta (FC) & FC $>0,70$ \\
\hline Validade Discriminante & VME versus & VME $>$ \\
\hline
\end{tabular}

Fonte: Adaptado de Marôco (2014).

padrão, indicando mais constância em relação aos demais questionamentos.

No que se refere ao Sistema I, destaca-se maior uniformidade nas respostas referente ao fato das pessoas responderem que acham que existem momentos nos quais se deve confiar em sua intuição, pelo fato de ter sido observado a maior média e menor desvio padrão.

\section{SISTEMAS INTUITIVO E RACIONAL}

A partir dos itens traduzidos e aplicados na amostra que versaram sobre a escala REl, buscou-se validar a pesquisa através de uma Análise Fatorial Exploratória. Decidiu-se por rotação oblíqua (Oblimin) dado que se espera um relacionamento entre as dimensões inerentes (HAIR, 2005).

Tal deliberação torna-se ainda mais importante uma vez que quando as atividades não demandam muita capacidade analítica, o sistema racional adota sugestões do intuitivo com pouca ou nenhuma modificação e, quando o inverso ocorre, o Sistema Intuitivo recorre ao Racional, visando um processamento mais detalhado e específico (KAHNEMAN, 20I2). O método de rotação selecionado possibilitou analisar se a troca de tarefas e informações entre os sistemas se configuraria como uma relação significativa entre ambos ou apenas um compartilhamento de informações, visando uma consolidação de tarefas independentes.

O número de fatores não foi fixa- do porque, embora Hodgkinson et al. (2009), Witteman et al. (2009) e Akinci e Sadler-Smith (20II) tenham encontrado 2 fatores, 0 instrumento foi originalmente concebido por Pacini e Epstein (1999) e validado para 4 fatores. Com isso, a falta de consistência no número de fatores encontrados na literatura internacional aliada ao fato da escala jamais ter sido validada para realidade brasileira culminou na decisão pela não-fixação de fatores. Os principais resultados oriundos da validação da escala encontram-se sumarizados na Tabela 4.

A escala pôde, então, ser validada com 3 fatores de significativa confiabilidade. Percebeu-se que, embora a intuição tenha se agrupado em um fator único, a racionalidade se dividiu em dois fatores. A matriz rotacionada, de padrão, encontra-se na Tabela 5 .

Os entrevistados pareceram perceber (o instrumento trata de percepção) a racionalidade como procedente de dois diferentes mecanismos: um lógico, Racionalidade I, e um mais profundo e exigente, Racionalidade 2. Essa relação é apresentada na Análise Fatorial Confirmatória do instrumento na Figura 3.

Os resultados do ajuste mantiveram-se dentro dos critérios estabelecidos para um bom ajuste do modelo, exceto no que tange ao $\chi 2$, o que já era esperado dado o tamanho da amostra. A validade do instrumento pode ser avaliada a partir da Tabela 6, onde o único problema encontrado seria a Validade Con- 
TABELA 2 - Resultados da análise descritiva

\begin{tabular}{|c|c|c|}
\hline \multirow{2}{*}{ Questões } & \multicolumn{2}{|c|}{ Componentes } \\
\hline & Média & Desvio Padrão \\
\hline REI_R1 & 2,5366 & 2,17806 \\
\hline REI_R2 & 2,8737 & 2,20915 \\
\hline REI_R3 & 6,9471 & 2,60111 \\
\hline REI_R4 & 3,5879 & 2,53979 \\
\hline REI_R5 & 2,3134 & 2,04892 \\
\hline REI_R6 & 6,2194 & 2,58036 \\
\hline REI_R7 & 2,3591 & 2,05441 \\
\hline REI_R8 & 3,0110 & 2,26109 \\
\hline REI_R9 & 2,8239 & 2,22256 \\
\hline REI_R10 & 5,0625 & 2,63395 \\
\hline REI_R11 & 3,7740 & 2,61311 \\
\hline REI_R12 & 4,8890 & 2,90438 \\
\hline REI_R13 & 5,6171 & 2,38022 \\
\hline REI_R14 & 6,1724 & 2,39311 \\
\hline REI_R15 & 5,4997 & 2,52123 \\
\hline REI_R16 & 6,9282 & 2,45579 \\
\hline REI_R17 & 7,0289 & 2,13610 \\
\hline REI_R18 & 2,5922 & 2,12698 \\
\hline REI_R19 & 7,0103 & 2,17022 \\
\hline REI_R20 & 7,9739 & 1,62202 \\
\hline REI_I1 & 6,4126 & 2,38621 \\
\hline REI_I2 & 3,6696 & 2,45539 \\
\hline REI_I3 & 5,6078 & 2,51783 \\
\hline REI_I4 & 6,2919 & 2,28672 \\
\hline REI_I5 & 5,3475 & 2,52451 \\
\hline REI_I6 & 5,4435 & 2,49098 \\
\hline REI_I7 & 4,9320 & 2,69222 \\
\hline REI_I8 & 5,3494 & 2,58596 \\
\hline REI_19 & 4,2477 & 2,47200 \\
\hline REI_I10 & 4,6613 & 2,63521 \\
\hline REI_I11 & 7,3774 & 1,92796 \\
\hline REI_I12 & 4,6456 & 2,73785 \\
\hline REI_I13 & 5,2120 & 2,78226 \\
\hline REI_I14 & 4,1371 & 2,60586 \\
\hline REI_I15 & 4,9166 & 2,47007 \\
\hline REI_I16 & 5,6776 & 2,49246 \\
\hline REI_I17 & 4,3061 & 2,31443 \\
\hline REI_I18 & 4,6722 & 2,62228 \\
\hline REI_I19 & 5,4507 & 2,54128 \\
\hline REI_I20 & 5,2303 & 2,26052 \\
\hline
\end{tabular}

Fonte: Elaboração própria. 
TABELA 4 - Resultados da validação da REI

\begin{tabular}{l|c}
\hline ASPECTO ANALISADO & COEFICIENTES \\
\hline Kayser-Meyer-OIkin (KMO) & 0,736 \\
\hline Comunalidade & {$[0,612 ; 0,807]$} \\
\hline Teste de Esferacidade de Bartlett & 7576,$393 ;$ p-valor $=0,000$ \\
\hline Medida de Adequação da Amostra (MSA) & {$[0,552 ; 0,849]$} \\
\hline Variância Explicada & $70,512 \%$ \\
\hline Alpha Fator 1 - Racionalidade Reflexiva & 0,692 \\
\hline Alpha Fator 2 - Racionalidade Lógica & 0,772 \\
\hline Alpha Fator 3 - Intuição & 0,783 \\
\hline
\end{tabular}

Fonte: Elaboração própria.

TABELA 5 - Matriz padrão da REI

\begin{tabular}{|c|c|c|c|}
\hline \multirow{2}{*}{ Questões } & \multicolumn{3}{|c|}{ Componente } \\
\hline & 1 & 2 & 3 \\
\hline $\begin{array}{l}\text { Eu tento evitar situações que requerem pensamentos muito profundos sobre } \\
\text { algo (REI_R1) }\end{array}$ & & ,841 & \\
\hline Eu não sou tão bom em entender problemas complicados (REI_R2) & & ,698 & \\
\hline Eu não gosto de ter que pensar muito (REI_R5) & & ,801 & \\
\hline $\begin{array}{l}\text { Eu sou muito melhor em entender coisas logicamente do que a maioria das } \\
\text { pessoas (REI_R13) }\end{array}$ & & & ,904 \\
\hline Eu tenho uma mentalidade lógica (REI_R14) & & & ,888 \\
\hline Eu gosto de confiar em minhas impressões intuitivas (REI_I1) & ,837 & & \\
\hline $\begin{array}{l}\text { Utilizar meus sentimentos instintivos normalmente funciona bem para eu resol- } \\
\text { ver problemas em minha vida (REI_I3) }\end{array}$ & ,849 & & \\
\hline Eu confio em minhas impressões (REI_I4) & ,851 & & \\
\hline Eu geralmente sigo meus instintos quando decido um curso de ação (REI_l6) & ,790 & & \\
\hline
\end{tabular}

Fonte: Dados da pesquisa.

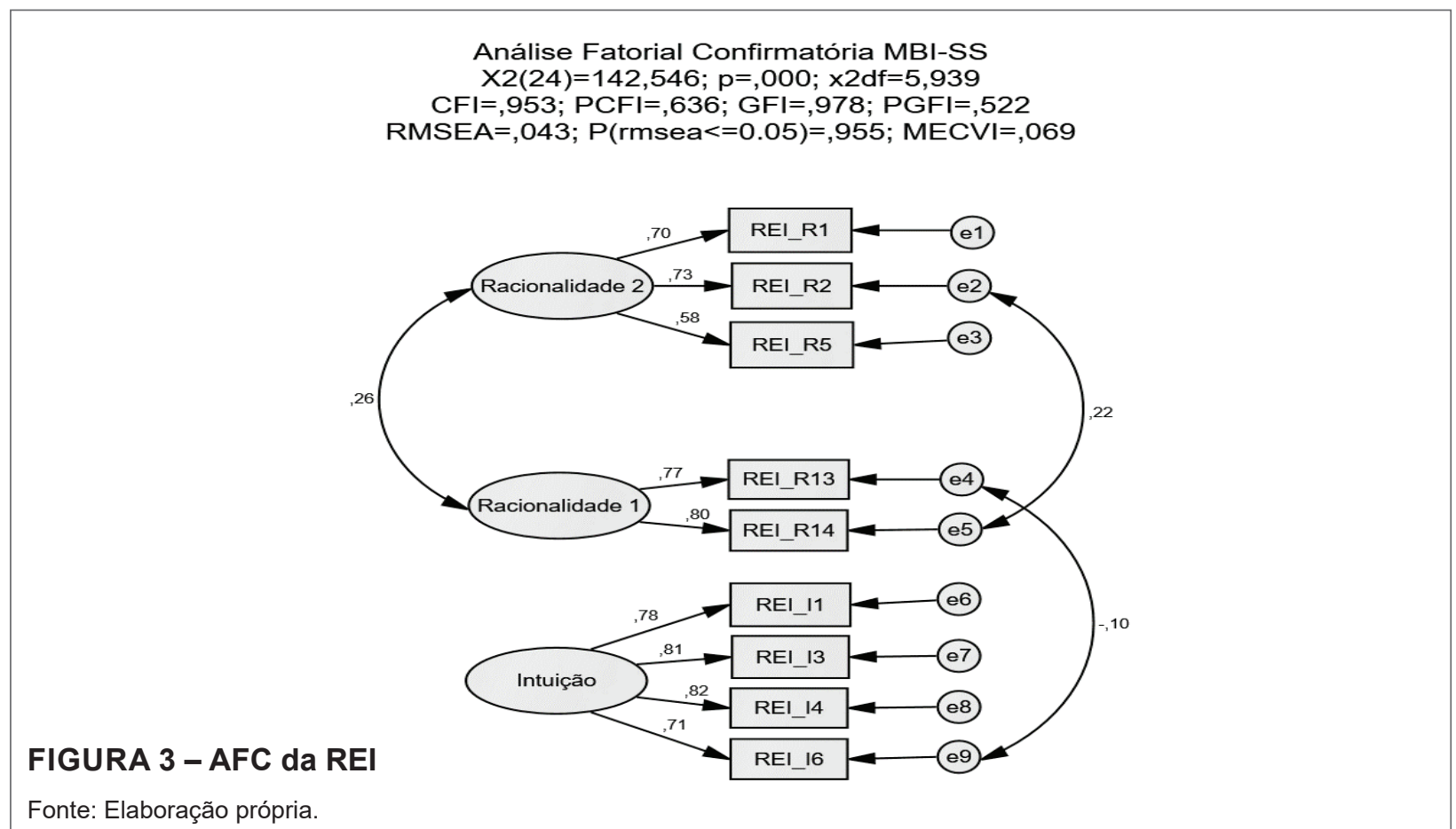


vergente e apenas no que tange ao VME da Racionalidade 2.

A percepção dos indivíduos acerca da racionalidade está em linha aos estudos de Stanovich (2009), Stanovich (20I I), Evans e Stanovich (20I3), Vaughan et al. (20I6) e Palkovics e Takac (2016). Nesses, a racionalidade é tratada como uma resultante de uma mentalidade algorítmica e reflexiva, ambas relacionadas. Entretanto, são vistas como diferentes níveis e independentes de um mesmo sistema.

Para Stanovich (2009), a mente reflexiva abrange a tendência individual de satisfação com respostas superficialmente atraentes, dado o desejo individual de finalizar o processo do pensamento. Sendo assim, pessoas que utilizam pouco sua mente reflexiva evitariam "pensamentos profundos", "problemas complicados" e "pensar muito". Esses aspectos se agruparam no fator Racionalidade 2 , que é denominado de "Racionalidade Reflexiva", sendo constitutivo do Sistema Racional.

A Racionalidade Reflexiva engloba a utilização plena da racionalidade no dia a dia a partir de questões que envolvem o "gostar" e "evitar". Diferentemente dessa, na Racionalidade I, tem-se uma abrangência estreita, onde analisa se o indivíduo "tem uma mentalidade lógica" e se é "melhor em entender coisas logicamente", não incluindo a prática da racionalidade, mas, sim, sua capacidade para tal. Tal aspecto corrobora com Stanovich (2009), onde a mente algorítmica estaria relacionada à capacidade de um indivíduo agir de forma racional, servindo de aparato para o processamento e as realizações de ações que permitem o indivíduo apresentarem crenças bem calibradas (nível reflexivo) e agirem adequadamente, visando atingirem seus objetivos (nível reflexivo).

Tem-se, ainda, que, para Kahneman (20I2), enquanto a mente reflexiva de Stanovich (2009) poderia ser tratada como a "racionalidade", a algorítmica seria a "inteligência". A Racionalidade 2 se aproxima da inteligência, uma vez que a lógica remonta ao raciocínio normativo, sendo comumente associada aos testes de inteligência e à capacidade cerebral, ambos relativos à mentalidade algorítmica.

Sendo assim, o fator Racionalidade 2 foi denominada de "Racionalidade Algorítmica" e estaria relacionado à mentalidade algorítmica devido aos dois aspectos: sua abrangência estreita (trata da racionalidade apenas como capacidade) e sua pertinência ao raciocínio normativo.

\section{CONCLUSÃO}

O presente trabalho se propôs a validar uma escala para mensuração dos Sistemas Cognitivos, aprofundando assim o entendimento de como estes se dividem. A necessidade de se entender os sistemas cognitivos se dá uma vez em que a literatura tende a analisar os sistemas a partir de uma estrutura bipartida (Sistema I e 2), contudo, a ideia de uma estrutura tripartida tem ganhado espaço e apoiadores

TABELA 6 - Testes de validade da REI

\begin{tabular}{l|c|c}
\hline Validade em análise & Fator & Resultados \\
\hline Validade Fatorial & - & $0,34 ; 0,67]$ \\
\hline \multirow{3}{*}{ Validade Convergente } & Racionalidade 2 & VME $=0,24$ e FC $=0,76$ \\
& Intuição & VME $=0,61$ e FC $=0,86$ \\
\cline { 2 - 3 } & Racionalidade 1 & VME $=0,45$ e FC $=0,71$ \\
\cline { 2 - 3 } Validade Discriminante & - & VME $>$ \\
\hline
\end{tabular}

Fonte: Elaboração própria. 
(STANOVICH, 2009; STANOVICH;WEST; TOPLAK 20II; EVANS; STANOVICH, 20I3;VAUGHAN et al., 2016; PALKOVICS; TAKAC, 2016), sobretudo na inteligência artificial (SAMUELS, 2005; STANOVICH, 2009; VAUGHAN, et al., 2016).

Ao testar-se a Validade de Constructo o único problema encontrado foi quanto a Validade Convergente e apenas no que tange aoVME da Racionalidade 2.A REl foi reconhecida estatisticamente encontrandose 3 fatores, dois relativos à racionalidade e um à intuição, havendo um relacionamento entre as duas racionalidades.

Tal subdivisão é similar à sugerida nos estudos de Stanovich (2009), Stanovich (20I I), Evans e Stanovich (20I3), Vaughan et al. (2016) e Palkovics e Takac (2016), onde a racionalidade é subdividida em uma algorítmica e outra reflexiva. Essa observação sugere uma necessidade de aprofundamento nas teorias da estrutura tripartida, até então parcamente explorada por pesquisadores que não são oriundos da Inteligência Artificial (SLOMAN; CHRISLEY, 2003; SAMUELS, 2005; STANOVICH, 2009; VAUGHAN, et al., 2016).

Por isso, acredita-se que se faz necessário o aprofundamento do estudo de tais relações, visando não apenas o entendimento de como os Sistemas Cognitivos afetam os vieses e heurísticas, mas também como esse relacionamento se diferencia dentre os diversos grupos. É importante salientar que a amostragem não foi aleatória, de forma que não se é possível a realização de generalizações. 


\section{REFERÊNCIAS}

AKINCI, Cinla; SADLER-SMITH, Eugene. Intuition in Management Research:A Historical Review. International Journal of Management Reviews, [s.I.], v. I4, n. I, p. 104-I 22, 14 jun. 201 I.Wiley-Blackwell. http://dx.doi.org//0.1 I I I/j. |468-2370.20| |.003|3.x.

BASEL, Jörn S.; BRÜHL, Rolf. Rationality and dual process models of reasoning in managerial cognition and decision making. European Management Journal, [s.l.], v. 3I, n. 6, p.745-754, dez. 20I3. Elsevier BV. http://dx.doi.org/10.1016/j.emj.2013.07.004.

CACIOPPO, John T.; PETTY, Richard $E$. The need for cognition. Journal of Personality And Social Psychology, [s.l.], v. 42, n. I, p.l I6I31, 1982.American Psychological Association (APA). http://dx.doi. org/10.1037/0022-35I4.42.1.116.

CARRUTHERS, Peter. The Architecture of the mind. Oxfort: Oxford University Press, 2006. 46 I p.

EPSTEIN, Seymour. Integration of the cognitive and the psychodynamic unconscious. American Psychologist, [s.I.], v. 49, n. 8, p.709724, 1994.American Psychological Association (APA). http://dx.doi. org// 0.1037/0003-066x.49.8.709.

EPSTEIN, Seymour et al. Individual differences in intuitive-experiential and analytical-rational thinking styles. Journal of Personality And Social Psychology, [s.l.], v. 7I, n. 2, p.390-405, 1996.American Psychological Association (APA). http://dx.doi.org// 0.1037/002235। 4.7।.2.390.

EVANS, Jonathan St B. T. Heuristic and analytic processes in reasoning. British Journal of Psychology, [s.I.], v. 75, n. 4, p.45I-468, nov. 1984. Wiley-Blackwell. http://dx.doi. org/ I 0. I I I I/j.2044-8295. I 984. tb01915.x.

EVANS, Jonathan St. B. T.; OVER, David E. Rationality and Reasoning. [s. L.]: Psychology Press, 1996. $181 \mathrm{p}$.

EVANS, Jonathan St.b.t.. In two minds: dual-process accounts of reasoning. Trends in Cognitive Sciences, [s.I.], v. 7, n. 10, p.454-459, out. 2003. Elsevier BV. http://dx.doi.org//0.1016/j. tics.2003.08.012.

EVANS, Jonathan St. B. T. Dual-Processing Accounts of Reasoning, Judgment, and Social Cognition. Annual Review of Psychology, [s.l.], v. 59, n. I, p.255-278, jan. 2008. Annual Reviews. http://dx.doi.org//0.1/46/ annurev.psych.59.103006.093629

EVANS, Jonathan St B T. Intuition and Reasoning:A Dual-Process Perspective. Psychological Inquiry, [s.I.], v. 2I, n. 4, p.3I3-326, 30 nov. 20I0. Informa UK Limited. http://dx.doi.org/I0.1080/1047840x.20I0.52I057.

EVANS, J. S. B. T.; STANOVICH, K. E. Dual-Process Theories of $\mathrm{Hi}$ gher Cognition: Advancing the Debate. Perspectives on Psychological Science, [s.l.], v. 8, n. 3, p.223-24I, I maio 2013. SAGE Publications. http://dx.doi. org//0.1 I 77//74569/6/2460685.

FRANKISH, Keith. Dual-Process and Dual-System Theories of Reasoning. Philosophy Compass, [s.I.], v. 5, n. 10, p.914-926, out. 2010. Wiley-Blackwell. http://dx.doi.org//0.1 I I I/j. 1747-999|.2010.00330.x.

HAIR, Joseph F. et al. Análise multivariada de dados. 5. ed. Porto Alegre: Bookman, 2005.

HAMMOND, Kenneth R. Human Judgment and Social Policy. New York: Oxford University Press, 1996.435 p.
HODGKINSON, Gerard P. et al. More than meets the eye? Intuition and analysis revisited. Personality and Individual Differences, [s.l.], v. 47, n. 4, p.342-346, set. 2009. Elsevier BV. http://dx.doi. org// 0.1016/j.paid.2009.03.025.

KAHNEMAN, Daniel. Rápido e Devagar: duas formas de pensar. Rio de Janeiro: Objetiva, 2012.610 p.

KAHNEMAN, Daniel. Maps of bounded rationality: a perspective on intuitive judgment and choice. 2002. PrizeLecture. Disponível em: <http://www.nobelprize. org/>. Acesso em: II mar. 2014.

KAHNEMAN, Daniel; FREDERICK, Shane. Representativeness revised: Attribute substitution in intuitive judgment. In: GILOVICH, T.; GRIFFIN, D.; KAHNEMAN, D. Heuristics of Intuitive Judgment: Extensions and Applications. New York: Cambridge University Press, 2002.

KAHNEMAN, D.; TVERSKY, A. An Analysis of Decision under Risk. Econometrica, v. 49, n. 2, p.263292, May. 1979.

KAHNEMAN, Daniel; TVERSKY, Amos. On the study of statistical intuitions. Cognition, [s.l], v. II, p. I23-I4I, 1982.

KAHNEMAN, Daniel; TVERSKY, Amos. Choices, values, and frames. American Psychologist, [s.l.], v. 39, n. 4, p.34I-350, 1984. American Psychological Association (APA). http://dx.doi.org/l0.1037/0003-066x.39.4.34I.

KEREN, Gideon; SCHUL, Yaacov. Two Is Not Always Better Than One:A Critical Evaluation of Two-System Theories. Perspectives on Psychological Science, [s.l.],v.4,n.6, p.533-550, nov. 2009. SAGE Publications. http://dx.doi.org/10.1 I I I/ j. 1745-6924.2009.0II 64.x.

LEVINSON, Stephen C. Interactio- 
nal biases in human thinking. Expressions and Implications of The Social Bias in Human Intelligence, [s.l.], p.22I-260, 1995. Cambridge University Press (CUP). http://dx.doi.org/10.1017/ cbo97805 I I62 I7l0.014.

MARÔCO, João. Análise de Equações Estruturais: Fundamentos Teóricos, Software \& Aplicações. 2. ed. Pêro Pinheiro: Report Number, 2014. 389 p.

PACINI, Rosemary; EPSTEIN, Seymour. The relation of rational and experiential information processing styles to personality, basic beliefs, and the ratio-bias phenomenon. Journal of Personality and Social Psychology, [s.l.], v. 76, n. 6, p.972-987, 1999.American Psychological Association (APA). http://dx.doi.org//0.1037//002235 | 4.76.6.972.

PALKOVICS, Michael Anton; TAKAC, Martin. Exploration of cognition-affect and Type I-Type 2 dichotomies in a computational model of decision making. Cognitive Systems Research, [s.l.], v. 40, p.144-160, dez. 2016. Elsevier BV. http://dx.doi. org/10.1016/j.cogsys.2016.06.00।

REBER,Arthur S. Implicit Learning and Tacit Knowledge. Journal of Experimental Psychology: General, [s. L.], v. I I8, n. 3, p.219-235, 1989.
SAMUELS, Richard. The complexity of cognition: Tractability arguments for massive modularity. In: CARRUTHERS, P.; LAURENCE, S.; STI$\mathrm{CH}, \mathrm{S}$. The innate mind. $\mathrm{Ox}$ ford: Oxford University Press, 2005. p. 107-121.

SINCLAIR, Marta. Handbook of Research Methods on Intuition. [s. L.]: Edward Elgar Pub, 20I4. 290 p.

SLOMAN, Steven A. The empirical case for two systems of reasoning. Psychological Bulletin, [s.l.], v. I 19, n. I, p.3-22, 1996. American Psychological Association (APA). http://dx.doi.org/10.1037/00332909.I19.1.3.

SLOMAN, Aaron; CHRISLEY, Ron. Virtual Machines and Consciousness. Journal of Consciousness Studies, [s. L.], v. 10, n. 4, p. I-40, 2003

STANOVICH, Keith E.; WEST, Richard F. Individual differences in reasoning: Implications for the rationality debate? Behavioral and Brain Sciences, [s.I.], v. 23, p.645-726, 2000.

STANOVICH, Keith E. The Robot's Rebellion: Finding meaning in the age of darwin. Chicago: University of Chicago Press, 2004. 358 p.

STANOVICH, Keith E. Distinguishing the reflective, algorithmic, and autonomous minds: Is it time for a tri-process theory? In: EVANS, Jonathan St. B. T.; FRANKISH, Keith. In Two Minds: Dual Processes and Beyond. [s. L.]: Oxford University Press, 2009. p. 55-88.

STANOVICH, Keith E..Rationality and the reflective mind. New York: Orxford University Press, $201 \mathrm{I}$.

STANOVICH, Keith E.;WEST, Richard F.;TOPLAK, Maggie E.The complexity of developmental predictions from dual process models. Developmental Review, [s.l.], v. 3 I, n. 2-3, p.103-I I8, set. 20 I I. Elsevier BV.

TVERSKY, A.; KAHNEMAN, D. Judment under uncertainty: Heuristics and biases. Science, v. 28, n. 4I57. p. II24- I I3 I, Sep. 1974.

VAUGHAN, Sandra L. et al. A dual-process Qualia Modeling Framework (QMF). Biologically Inspired Cognitive Architectures, [s.l.], v. 17, p.7l-85, jul. 2016. Elsevier BV. http://dx.doi. org/10.1016/j.bica.2016.07.00I.

WITTEMAN, Cilia et al. Assessing Rational and Intuitive Thinking Styles. European Journal of Psychological Assessment, [s.l.], v. 25, n. I, p.39-47, jan. 2009. Hogrefe \& Huber. http://dx.doi. org/I0.1027/1015-5759.25.1.39. 